\title{
Cell cycle-regulated histone acetylation required for expression of the yeast HO gene
}

\author{
Jocelyn E. Krebs, ${ }^{1}$ Min-Hao Kuo, ${ }^{2}$ C. David Allis, ${ }^{2}$ and Craig L. Peterson ${ }^{1,3}$ \\ ${ }^{1}$ Program in Molecular Medicine and Department of Biochemistry and Molecular Biology, University of Massachusetts \\ Medical Center, Worcester, Massachusetts 01605 USA; ${ }^{2}$ Department of Biochemistry, University of Virginia Medical School, \\ Charlottesville Virginia 22906 USA
}

\begin{abstract}
Expression of the yeast $\mathrm{HO}$ gene in late $\mathrm{G}_{1}$ of the cell cycle requires the SWI/SNF chromatin remodeling complex, the Gcn5p histone acetyltransferase, and two different sequence-specific transcriptional activators, Swi5p and Swi4p/Swi6p. We have used chromatin immunoprecipitation assays to investigate the role of each of these trans-acting factors in establishing a cell cycle-regulated domain of histone acetylation surrounding the $\mathrm{HO}$ upstream regulatory region. We detect a $\sim 1-\mathrm{kb}$ domain of $\mathrm{H} 3$ and $\mathrm{H} 4$ acetylation that is established in mid- $\mathrm{G}_{1}$, prior to and independent of $\mathrm{HO}$ transcription, which then declines with kinetics similar to inactivation of $\mathrm{HO}$. This cell cycle burst of histone acetylation requires Gcn5p, SWI/SNF, and the Swi5p activator, but occurs in the absence of the Swi4p activator. We also find that inactivation of the Sin $3 p / R p d 3 p$ deacetylase complex leads to a high level of acetylation at the $\mathrm{HO}$ locus throughout the cell cycle. We propose a sequential model for activation of $\mathrm{HO}$ in which the Swi5p-dependent recruitment of the Gen5p acetyltransferase requires chromatin remodeling events by the SWI/SNF complex.
\end{abstract}

[Key Words: Cell cycle; yeast; HO gene; histone acetylation; chromatin remodeling]

Received March 30, 1999; revised version accepted April 13, 1999.

Transcriptional activation in eukaryotes requires that transcription factors gain access to their target genes in a chromatin environment. The wrapping of DNA around histone proteins to form nucleosomes, as well as the higher-order folding of nucleosomal arrays, produce structures that are inaccessible to the transcription machinery. Naturally, eukaryotes have evolved mechanisms with which to contend with these repressive chromatin structures (Workman and Kingston 1998).

In recent years a large number of protein complexes capable of modifying chromatin structure have been identified. These chromatin remodelers fall into two general classes: the ATP-dependent remodelers and the histone acetyltransferases (HATs). The prototype of the ATP-dependent class of remodeling enzymes is the SWI/ SNF complex of Saccharomyces cerevisiae, which is required for the transcriptional activation of a subset of genes and for the full functioning of several transcriptional activators (Laurent and Carlson 1992; Laurent et al. 1993; Peterson et al. 1994; Peterson 1996; Burns and Peterson 1997; Ryan et al. 1998). In vitro, SWI/SNF uses the energy of ATP hydrolysis to alter DNA-histone con-

${ }^{3}$ Corresponding author.

E-MAIL craig.peterson@ummed.edu; FAX (508) 856-4289. tacts to make nucleosomal DNA more accessible to transcription factors (Cote et al. 1994, 1998; Imbalzano et al. 1994, 1998; Utley et al. 1996) and restriction enzymes (Logie and Peterson 1997). Yeast have also provided the first example of a transcription-related HAT, Gcn5 (Brownell et al. 1996), which is a member of several multisubunit complexes (Grant et al. 1997; Pollard and Peterson 1997; Saleh et al. 1997; Eberharter et al. 1998). GCN5-dependent HAT complexes acetylate nucleosomal histones and can promote transcription from nucleosomal templates in vitro (Steger et al. 1998; Utley et al. 1998; Ikeda et al. 1999). In vivo, GCN5 is required for expression of a set of genes that overlaps with those that require SWI/SNF; in fact, gcn5 swi/snf double mutants exhibit synthetic phenotypes (Pollard and Peterson 1997; Roberts and Winston 1997). Residues in GCN5p that are critical for HAT activity in vitro are also required for Gcn5p's transcriptional activation function in vivo (Candau et al. 1997; Wang et al. 1998), and GCN5 has been shown to be required for promoter-directed acetylation at the HIS3 gene in vivo (Kuo et al. 1998). Although Gcn5p-containing HAT complexes have been shown to interact with several activator proteins in vitro (Utley et al. 1998), little is known about how these chromain remodelers are targeted to their sites of action in vivo. 
One of the yeast genes whose expression requires both Gcn5p and SWI/SNF is HO (Pollard and Peterson 1997; Perez-Martin and Johnson 1998), which encodes a sitespecific endonuclease that initiates the process of mating-type interconversion. $\mathrm{HO}$ expression is tightly regulated by cell type and during the cell cycle, being expressed in only a small window in late $G_{1}$ and only in haploid mother cells /cells which have budded previously) (for review, see Herskowitz et al. 1992; Nasmyth 1993). This complex pattern of $H O$ expression requires an unusually large upstream regulatory region ( 2000 $\mathrm{bp})$, and a host of positive and negative trans-acting factors, including SWI/SNF, Gcn5p, the sequence-specific activators Swi5p and Swi4p/Swi6p, the Sin3p/Rpd3p deacetylase complex, and the daughter-specific repressor, Ash1p (Breeden and Nasmyth 1987; Sternberg et al. 1987; Herskowitz et al. 1992; Nasmyth 1993; Jansen et al. 1996; Sil and Herskowitz 1996). Swi5p is involved in mother/daughter regulation and binds to two sequence elements located 1300- and 1800-bp upstream from the HO mRNA start site. The SWI4 and SWI6 genes encode two subunits of a transcriptional activator, called SBF, that binds in late $\mathrm{G}_{1}$ to 10 cell cycle boxes located within a 900-bp region upstream of the $H O$ mRNA start site. Binding of SBF in late $G_{1}$ is not sufficient for activation of $H O$, as a subsequent step requires phosphorylation by a Cdc28p/Clnp kinase at the $\mathrm{G}_{1} / \mathrm{S}$ boundary (Start) (Harrington and Andrews 1996; Koch et al. 1996). The inactivation of $\mathrm{HO}$ expression after Start requires the activity of an S-phase Cdc28p/Clbp kinase (Amon et al. 1993).

In this study, we have examined the histone acetylation pattern of the $H O$ promoter during G1 and early S phase. By taking advantage of mutations in the various regulators of $H O$, we have determined the activator and remodeler dependence of the changes in histone acetylation. Our results show that there is a cell cycle-regulated wave of GCN5-dependent histone acetylation that is restricted to $\sim 1 \mathrm{~kb}$ of the $H O$ upstream regulatory region encompassing the 10 cell cycle boxes and the TATA element. GCN5-dependent acetylation requires Swi5p and SWI/SNF, but occurs in the absence of Swi4p and precedes transcription. In contrast, inactivation of the $\operatorname{Sin} 3 p / R p d 3 p$ deacetylase complex leads to an expanded domain of acetylation as well as high levels of histone acetylation throughout the cell cycle. We propose a sequential order of action for the regulators of $\mathrm{HO}$ expression in which the Swi5p activator recruits SWI/ SNF whose remodeling activity then targets a Gcn5pcontaining HAT complex to the promoter. Acetylation of nucleosomes that encompass the cell cycle boxes may then facilitate binding of the Swi4p/Swi6p complex, which acts very late in the activation cycle. And finally, the $\operatorname{Sin} 3 p / R p d 3 p$ deacetylase complex may play a significant role in erasing acetylation events, ensuring that the HO locus has a low level of histone acetylation prior to the beginning of the next cell cycle. These data support the view that chromatin remodeling factors are recruited by site-specific activators, and suggest that two different classes of chromatin remodelers may function at separate and successive steps in gene activation.

\section{Results \\ Nucleosomes encompassing the HO promoter are dynamically acetylated in a cell cycle- and GCN5-dependent manner}

Expression of the $\mathrm{HO}$ gene is restricted to a short window in late $\mathrm{G}_{1}$ of mother cells. $\mathrm{HO}$ expression is repressed in daughter cells by the repressor protein, Ash1p, and thus inactivation of $A S H 1$, allows expression of $H O$ in both mothers and daughters. Importantly, a deletion of the ASH1 gene does not disrupt other modes of $H O$ regulation, such as haploid specificity and cell cycle regulation. Therefore, we have used an isogenic set of strains for our analyses that harbor a deletion of ASH1, allowing us to obtain homogeneous cell populations in which $H O$ is uniformly expressed in late $G_{1}$ of the cell cycle. To examine acetylation events throughout $\mathrm{G}_{1}$ and early $S$ phase, we synchronized cells in $G_{2} / M$ with nocodazole, and then washed out the nocodazole to allow the cells to progress through a synchronous cell cycle. Cell aliquots were taken at 15-min intervals following release from the nocodazole block, and these samples were analyzed for position in the cell cycle (by budding index), HO expression (by primer extension of RNA), and histone acetylation [by chromatin immunoprecipitation (ChIP)]. Figure 1A shows a typical nocodazole arrest/release timecourse for a GCN5 ash1 strain, showing budding index and $\mathrm{HO}$ expression. After $3 \mathrm{hr}$ of incubation with nocodazole, $\sim 100 \%$ of the cells are arrested in the $\mathrm{G}_{2} / \mathrm{M}$ phase of the cell cycle as indicated by the large budded phenotype (time zero). Cells begin to enter $G_{1}$ (cells with no buds) between 30 and 45 min after release from the nocodazole block, and cells traverse the $G_{1} / S$ boundary $\sim 90 \mathrm{~min}$ after release (appearance of small buds). At $120 \mathrm{~min}$ after release, most cells have entered $S$ phase as indicated by the high proportion of budded cells. As expected, $H O$ transcripts are first detected by primer extension at 75-90 min after release, immediately before the onset of $\mathrm{S}$ phase at $90 \mathrm{~min}$ (Fig. 1A).

GCN5 ash1 and gcn5 ash1 cells were synchronized with nocodazole, timepoints were fixed with formaldehyde, and chromatin was isolated and processed for immunoprecipitation as described in Materials and Methods. To investigate the pattern of histone acetylation at the HO promoter, fragmented chromatin was immunoprecipitated with antibodies specific to a diacetylated form of histone H3 acetylated at lysines 9 and 14 $(\alpha$ H3ac.9/14). These lysines are the preferred sites of acetylation by Gcn5p in vitro (Kuo et al. 1996), and a mutation in $\mathrm{H} 3$ that changes lysine 14 to arginine confers a strong, synthetic growth defect in gcn5 cells (Zhang et al. 1998). Immunoprecipitated chromatin and the input material were deproteinized and applied to slot blots, which were then hybridized with either total genomic DNA, rDNA, or a DNA probe specific for the $H O$ TATA region (see Fig. 2A). PhosphorImager scans of representative slot blots probed with the HO TATA probe are shown in Figure 1B. Quantitation of these blots as well as blots probed with genomic or rDNA is shown in Figure 1C. Quantitated data (Fig. 1C) are presented as IP 

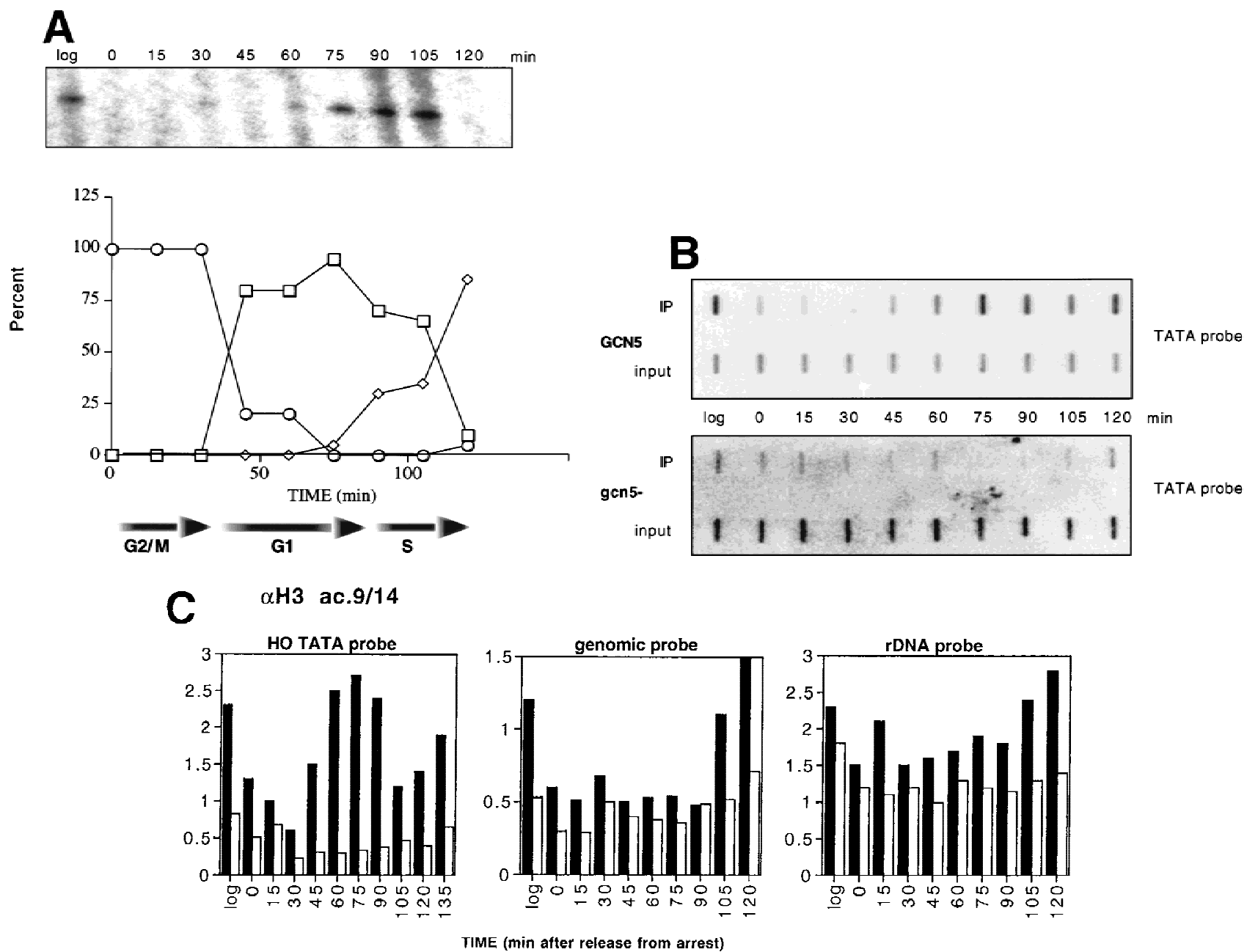

TIME (min after release from arrest)
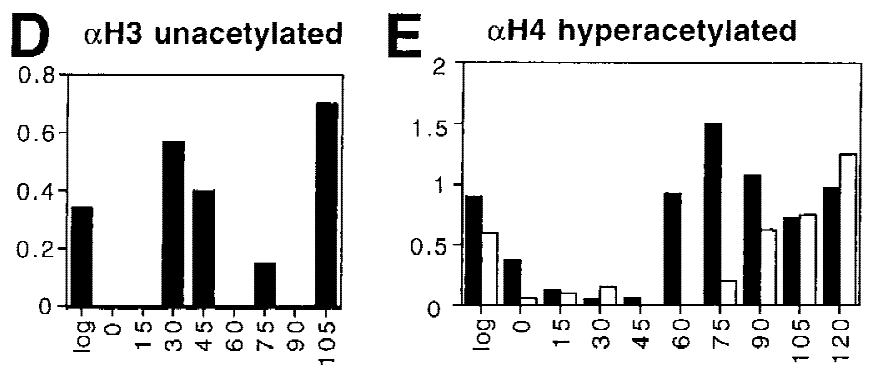

Figure 1. Nucleosomes at the TATA region of $H O$ are subject to GCN5-dependent, transient acetylation prior to transcription. $(A)$ Budding index and primer extension analysis of $H O$ transcripts show the cell cycle-dependence of $H O$ expression. Cells were arrested in $\mathrm{G}_{2} / \mathrm{M}$ by treatment with nocodazole, as described in Materials and Methods. Percentages of cells with no buds $(\square)\left(\mathrm{G}_{1}\right)$, small buds $(\diamond)$ (S phase), or large buds $(O)\left(G_{2} / M\right)$ were determined at the times shown after release from nocodazole arrest. Samples were taken from these timepoints for either RNA analysis or for ChIPs (see below). $H O$ transcripts are detectable by primer extension in late $\mathrm{G}_{1}$ and peak at the onset of S phase (appearance of small buds; 90-105 min in wild-type cells). (B) Results of ChIP assays for either wild-type or gcn5- cells. Chromatin obtained from cells released from nocodazole arrest as described above was immunoprecipitated with antibodies that recognize histone $\mathrm{H} 3$ acetylated at positions 9 and $14(\alpha \mathrm{H} 3 \mathrm{ac} .9 / 14)$. DNA obtained from either input or immunoprecipitated material was applied to slot blots. Representative slot blots probed for the HO TATA region are shown. (C) Quantitation of representative slot blots. Blots were quantitated with a PhosphorImager; the results are expressed as the ratio of the bound to the input material (IP efficiency). The graph at left shows the results for the HO TATA probe for either $G C N 5^{+}$or $g c n 5^{-}$cells, showing the GCN5-dependent peak of acetylation between 60 and $90 \mathrm{~min}$ after release from nocodazole arrest. The high levels of acetylation detected in log phase cells, as well as in early S phase, are also partially GCN5 dependent. The middle graph depicts the global levels of GCN5-dependent acetylation using the genomic DNA probe, and acetylation detected by the rDNA probe is shown at

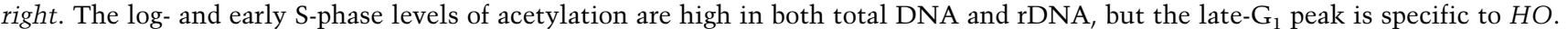
Data shown are representative of four separate experiments with ChIP extracts from two independent cell synchronizations. $(D)$ ChIP analysis of the wild-type samples from $C$ using antibodies against unacetylated $\mathrm{H} 3$. The smaller scale on this graph reflects the weaker IP efficiency of these antibodies. (E) ChIP analysis of wild-type and gcn5- cells with antibodies recognizing hyperacetylated histone H4. (Solid bars) Gen5p; (open bars) gen5. 


\section{$\mathrm{A}^{\text {HO PROMOTER }}$}
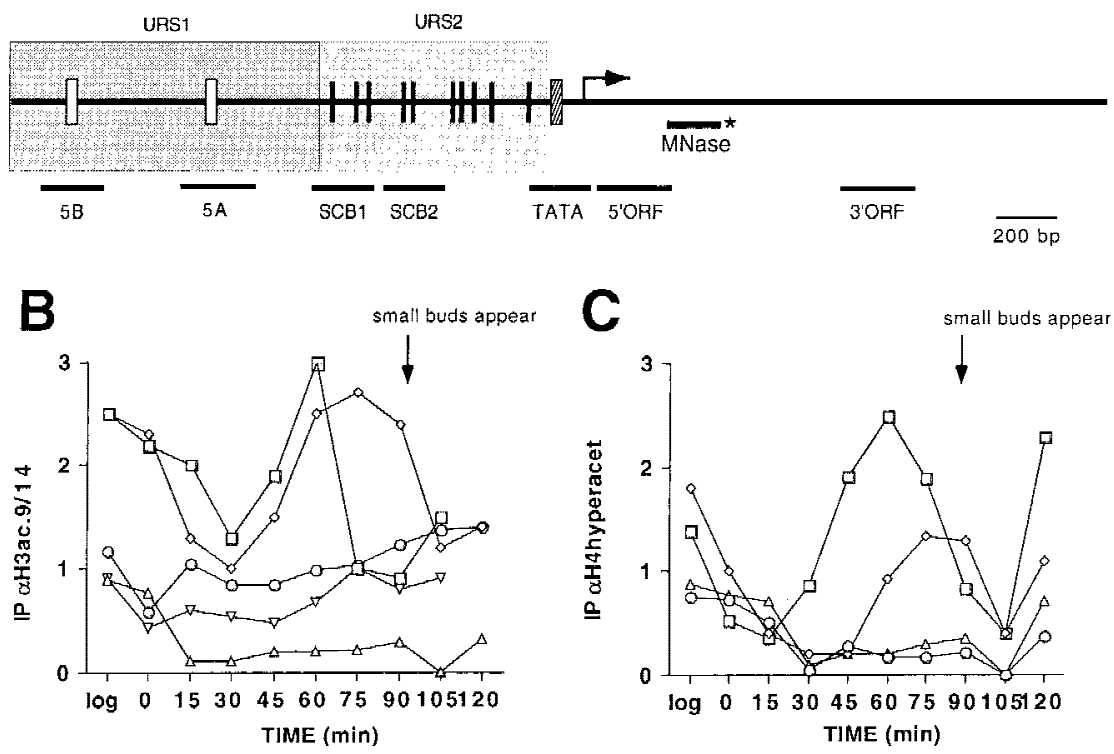

Figure 2. Acetylation at the $H O$ promoter is limited to a $1-\mathrm{kb}$ region encompassing the TATA box and the Swi4p/Swi6p-binding sites. $(A)$ Schematic of the $H O$ promoter showing the probes used in this study. (Hatched bars) TATA; (solid bars) SWI cell cycle box (SCB); (open bars) Swi5p binding site. (B) Results of probe scan of $\mathrm{HO}$ for samples precipitated with $\alpha \mathrm{H} 3 \mathrm{ac} .9 / 14$. The slot blots shown in Fig. 1B were stripped and hybridized with a variety of DNA probes covering the $\mathrm{HO}$ promoter and coding region. The results for the TATA probe are the same as shown in Fig. 1C. Probes for the Swi4p/Swi6p-binding sites (SCB1 and SCB2) also detect a peak of acetylation that occurs slightly before the peak detected at the TATA. Probes for either of the Swi5pbinding sites (5A and $5 \mathrm{~B})$ or for the $H O$ coding region (5' ORF or $3^{\prime}$ ORF) do not detect a peak of acetylation. $(C)$ Results of probe scan of $H O$ for samples precipitated with antibodies to hyperacetylated $H 4 .(B, C)(\triangle)$ 5' ORF; $(\diamond)$ TATA; $(\square)$ SCB1; (O) 5B; ( $\nabla) 5 \mathrm{~A}$. efficiencies, in which the signal obtained from the immunoprecipitated material is normalized to the input sample. In the GCN5 ash1 cells, there is a strong, transient peak of $\mathrm{H} 3$ acetylation that begins 45 min after release from the nocodazole block and declines between 90 and $105 \mathrm{~min}$ (Fig. 1C, left). This peak of acetylation precedes $H O$ transcription by $\sim 15-30 \mathrm{~min}$ (Fig. 1A) and declines with kinetics similar to the disappearance of $H O$ transcripts (Fig. 1A). Furthermore, $\mathrm{G}_{1}$ acetylation of the $H O$ locus is absent in gcn 5 ash 1 cells (open bars), and it does not occur at the rDNA locus (right) or throughout the yeast genome (center). Thus, histone $\mathrm{H} 3$ that is assembled into nucleosomes that encompass the $\mathrm{HO}$ TATA element is dynamically acetylated in early $\mathrm{G}_{1}$ by a GCN5-dependent acetyltransferase.

Gcn5p can also acetylate histone $\mathrm{H} 4$ in vitro (Kuo et al. 1996), and substitution of lysines within the H4 amino-terminal domain also show synthetic phenotypes with gcn5 mutants in vivo (Zhang et al. 1998). To investigate histone $\mathrm{H} 4$ acetylation at the $\mathrm{HO}$ locus during $\mathrm{G}_{1}$ and early $S$ phase, we immunoprecipitated fixed chromatin samples with antibodies that recognize hyperacetylated histone $\mathrm{H} 4$, and immunoprecipitated material was detected with the HO TATA probe. As shown in Figure $1 \mathrm{E}$, there is a transient peak of $\mathrm{H} 4$ acetylation that coincides with the peak observed for H3 (60-90 min postrelease). Furthermore, this peak of $\mathrm{H} 4$ hyperacetylation is also GCN5 dependent (open bars).

If $\mathrm{HO}$ chromatin is dynamically acetylated during $\mathrm{G}_{1}$, then we should expect that the level of unacetylated histone $\mathrm{H} 3$ would show a reverse pattern, declining in $\mathrm{G}_{1}$. To test this possibility, we immunoprecipitated a subset of the same chromatin samples used in Figure 1C using antibodies directed against unacetylated histone $\mathrm{H} 3$ (Fig. 1D). As predicted, and in contrast to our results obtained with antibodies to acetylated histones, the level of unacetylated histone $\mathrm{H} 3$ at the $\mathrm{HO}$ locus is high in late anaphase/early $\mathrm{G}_{1}(30 \mathrm{~min})$, and decreases as cells enter (45 $\mathrm{min}$ ) and traverse $\mathrm{G}_{1}(75 \mathrm{~min})$. Thus, the immunoprecipitation profile for unacetylated $\mathrm{H} 3$ does not show a $G_{1}$ peak, but is the reciprocal of that seen for acetylated histones.

\section{GCN5 contributes to genome-wide acetylation} of histone H3 during $S$ phase

We also routinely observe an additional increase in $\mathrm{H} 3$ acetylation as cells enter S phase (Fig. 1C, 105-120 min). This second peak of $\mathrm{H} 3$ acetylation is most apparent with the genomic and rDNA probes, and it is partially dependent on GCN5 (right and center, respectively). The hyperacetylation of histone $\mathrm{H} 4$ also increases dramatically in $\mathrm{S}$ phase, but in this case the increase does not depend on GCN5 (Fig. 1E). A role for Gen5p in S-phase $\mathrm{H} 3$ acetylation may also provide an explanation for the partial GCN5 dependence of genome-wide acetylation observed in chromatin isolated from logarithmically growing cells (Fig. 1, bars marked $\log _{\text {; }}$ see also Kuo et al. 1998). Thus, whereas the $\mathrm{G}_{1}$ peak of $\mathrm{H} 3$ and $\mathrm{H} 4$ acetylation is specific for the $\mathrm{HO}$ locus, Gen $5 \mathrm{p}$ also appears to contribute to a global acetylation of histone $\mathrm{H} 3$ during $\mathrm{S}$ phase.

$G_{1}$-specific acetylation at the $\mathrm{HO}$ locus is restricted to a $1-k b$ region of upstream regulatory sequences

Previous studies have shown that GCN5 is required for H3 acetylation surrounding the HIS3 gene, and that 
these acetylation events may be restricted to only $\sim 200$ bp of upstream sequences (i.e., one to two nucleosomes; Kuo et al. 1998; M.H. Kuo and C.D. Allis, unpubl.). We investigated the extent of $\mathrm{H} 3$ and $\mathrm{H} 4$ acetylation at the $H O$ locus by using hybridization probes that span the entire $\mathrm{HO}$ locus (Fig. 2A). In addition to the TATA probe described above, we generated probes against each of the Swi5p-binding sites (Fig. 2A), against regions containing several of the Swi4p/Swi6p-binding sites (SCB1 and SCB2), as well as probes for the coding region of $H O$ (5'ORF and 3'ORF). We also synthesized probes to the coding regions of two genes that surround the $\mathrm{HO}$ locus, SSB1 and GCS1 (not shown). Slot blots such as those shown in Figure 1 were then sequentially hybridized with these nine DNA probes. A subset of these results is shown in Figure 2B for H3 diacetylation and Figure 2C for $\mathrm{H} 4$ hyperacetylation.

The probe scan provided two important results. First, $\mathrm{H} 3$ and $\mathrm{H} 4$ acetylation at the $H O$ locus during $\mathrm{G}_{1}$ is restricted to $\sim 1 \mathrm{~kb}$ of upstream regulatory sequences that encompasses the TATA box (TATA probe) and all of the Swi4p/Swi6p-binding sites (SCB1 and SCB2 probes) (Fig. $2 \mathrm{~B}$ and data not shown). MNase digestion mapping indicates that this region contains at least six nucleosomes (J. Krebs and C.L. Peterson, unpubl.). The $G_{1}$ peak of acetylation is not observed either upstream of this region (Swi5p-binding sites A and B) or in the coding region of HO (ORF probes) (Fig. 2; data not shown). Acetylation is also low in the coding regions of genes that flank $\mathrm{HO}$ (data not shown). Second, we repeatedly observe acetylation at the distal Swi4p/Swi6p-binding sites (SCB probes) earlier in the cell cycle than that seen at the TATA region (peak at $60^{\prime}$ for SCB probes vs. peak at $75^{\prime}$ for TATA probe). This is consistent with an acetyltransferase being targeted to the far upstream region of the HO promoter, which then progresses downstream toward the TATA element, creating a wave of histone acetylation.

\section{Acetylation of histone $\mathrm{H} 3$ at the $\mathrm{HO}$ promoter requires Swi5p and the SWI/SNF complex}

Next, we wished to determine whether the recruitment or activity of the GCN5-dependent HAT might require the Swi5p or Swi4p site-specific transcriptional activators or the chromatin remodeling activity of the SWI/ SNF complex. We therefore constructed an isogenic set of ash1s strains that also carried a deletion of either SWI2 (which encodes the ATPase subunit of SWI/SNF), SWI5, or SWI4. Synchronous populations of each of these strains were generated by nocodazole arrest and release, and histone $\mathrm{H} 3$ acetylation at the $\mathrm{HO}$ TATA region was analyzed by ChIP (Fig. 3). In a swi5 ash1 double mutant, $\mathrm{H} 3$ acetylation at the $H O$ locus is not established during $\mathrm{G}_{1}$, although acetylation at $H O$ and throughout the genome still increases at $\mathrm{S}$ phase (Fig. 3A and data not shown). Likewise, there is no significant peak of $\mathrm{H} 3$ acetylation in swi2 ash1 cells, whereas overall acetylation in log and $\mathrm{S}$ phase shows wild-type levels (Fig. 3A; data not shown). SWI5 and SWI2 are also re-
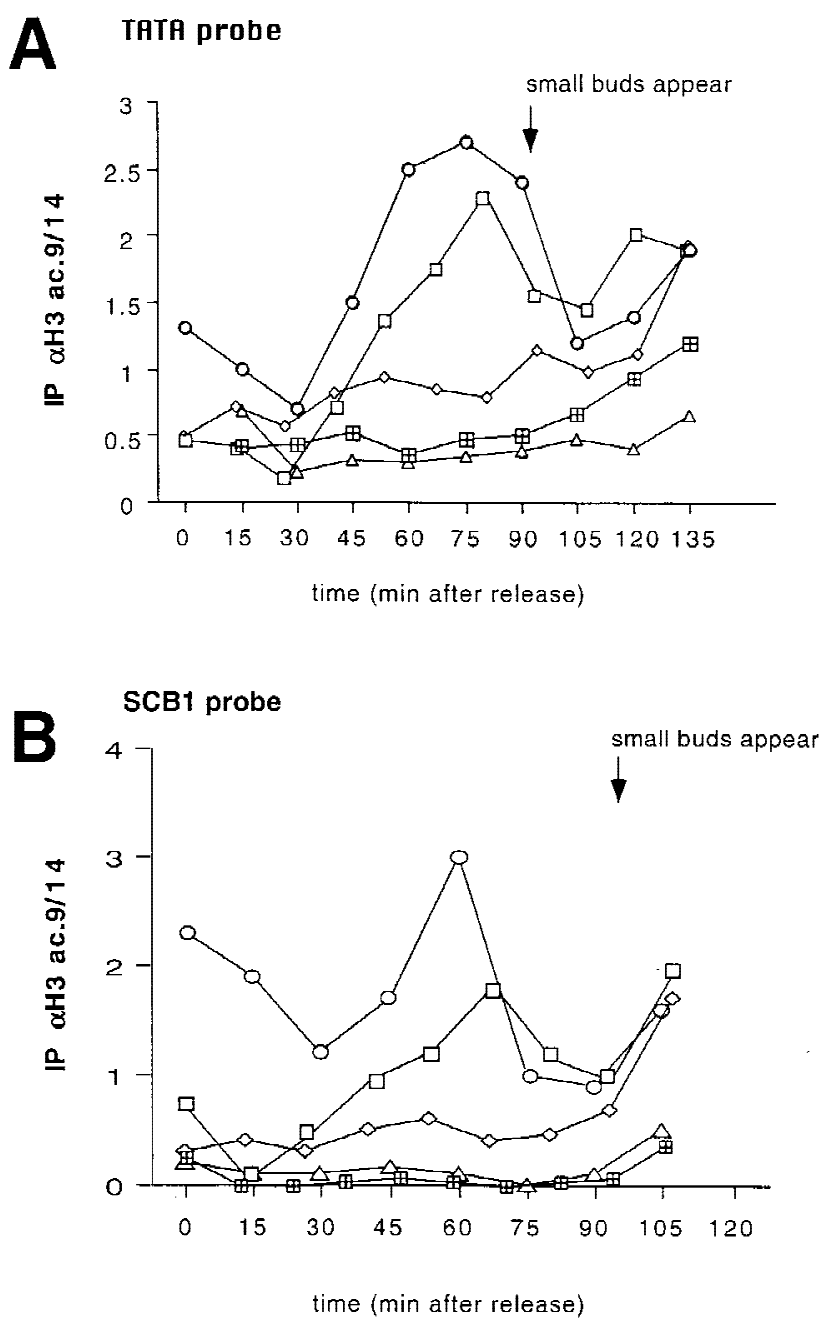

Figure 3. The cell cycle-regulated domain of histone $\mathrm{H} 3$ acetylation at $H O$ requires Gcn5p, Swi5p, and an intact SWI/SNF complex. SWI+, swi2-, swi5-, or swi4- cells were synchronized with nocodazole and samples were fixed for chromatin IP after synchronous release. ChIPs were performed with the antibody to diacetylated histone $\mathrm{H} 3$ (ac.9/14). The swi4 and swi2 mutants showed a significant delay in recovering from nocodazole arrest and thus synchronies are aligned by the time of bud emergence (arrow at top). Time scale shown on the $\mathrm{x}$ axis is from the SWI+ synchrony. Data shown are the result of four separate experiments from each strain with two independent cell synchronies. (A) Acetylation at the $H O$ TATA region in wild-type $(\mathrm{WT})(\bigcirc)$ cells and in swi5 $(\boxplus)$, swi2 $(\diamond)$, swi4 $(\square)$, and gcn5 $(\triangle)$ mutants. (B) Acetylation over the SCB elements in wild-type cells and in swi5, swi2, swi4, and gcn5 mutants.

quired for acetylation events outside the HO TATA box, as $\mathrm{H} 3$ acetylation over the SCB1 region also requires Swi5p and an intact SWI/SNF complex (Fig. 3B). Thus, recruitment or activity of a GCN5-dependent histone acetyltransferase requires one sequence-specific activator, Swi5p, and the SWI/SNF chromatin remodeling complex.

Regulated expression of $\mathrm{HO}$ relies on the combined 
action of two distinct transcriptional activators, Swi5p and Swi4p/Swi6p. Whereas H3 acetylation requires the Swi5p activator, we observe a normal peak of $\mathrm{H} 3$ acetylation at both the HO TATA and the SCB1 regions in a swi4 ash1 double mutant (Fig. 4A,B). Thus, these data indicate that the Swi4p/Swi6p activator may function at a step that is subsequent to the GCN5-dependent acetylation of the $H O$-upstream regulatory region. It is also important to note that there is no $H O$ transcription in a swi4 mutant (Stern et al. 1984; Breeden and Nasmyth 1987; J.E. Krebs, and C.L. Peterson, unpubl.) and we can therefore conclude that acetylation not only appears to precede transcription in wild-type cells, it can also occur in the complete absence of transcription.

\section{Role of the Sin3p/Rpd3p deacetylase complex}

Although the data presented in Figure 3 identifies the trans-acting factors required for establishing cell cycleregulated acetylation at the $H O$ locus, these results do not address how these acetylation events are eliminated when $\mathrm{HO}$ is repressed in early $\mathrm{S}$ phase or how the deacetylated state is established as cells enter $\mathrm{G}_{1}$. For instance, we find that the levels of acetylated histones $\mathrm{H} 3$ and $\mathrm{H} 4$ decline precipitously in early $\mathrm{S}$ phase just prior to the inactivation of $\mathrm{HO}$ transcription (Figs. 1-3). Likewise, at the start of $\mathrm{G}_{1}, \mathrm{H} 3$ acetylation at the $\mathrm{HO}$ locus is much lower than the levels observed throughout the genome (Figs. $1 \mathrm{C}$ and $2 \mathrm{~B}$ ). One possibility is that these deacetylation events might be controlled by the Sin3p/Rpd3p deacetylase complex (Kasten et al. 1997), because SIN3 (SDI1) and RPD3 (SDI2) were identified as negative regulators of $\mathrm{HO}$ expression (Nasmyth et al. 1987; Sternberg et al. 1987; Stillman et al. 1994). To address this possibility, we constructed a $\sin 3$ ash 1 double mutant, and a synchronous cell population was generated by nocodazole arrest and release. Histone $\mathrm{H} 3$ acetylation at the $H O$ locus was then analyzed during the cell cycle by ChIP (Fig. 4). Whereas wild-type cells exhibit a dramatic burst of histone acetylation as cells enter $\mathrm{G}_{1}$, inactivation of SIN3 leads to a high level of H3 acetylation that shows little cell cycle periodicity (Fig. 4A). In particular, $\mathrm{H} 3$ acetylation does not decline at the $\mathrm{G}_{1} / \mathrm{S}$ boundary, but rather the level of $\mathrm{H} 3$ acetylation increases further as cells enter S phase. Furthermore, H3 acetylation is also high in the $H O$ coding region and upstream of the SCB elements (Fig. 4B,C). Although histone deacetylation in early $\mathrm{S}$ phase seems to parallel inactivation of $\mathrm{HO}$ expression in wild-type cells, constitutive histone acetylation does not alter the cell cycle timing of $\mathrm{HO}$ activation (J.E. Krebs and C.L. Petersen, data not shown; see also Nasmyth et al. 1987). Thus, inactivationof the $\operatorname{Sin} 3 p / R p d 3 p$ deacetylase complex disrupts the size of the acetylated domain and eliminates the cell cycle-regulated acetylation of the $H O$ upstream region. The cell cycle timing of $H O$ expression, however, appears to be primarily controlled by the regulation of Swi4p/Swi6p by Cdc28p/cyclin kinases (Harrington and Andrews 1996; Koch et al. 1996).
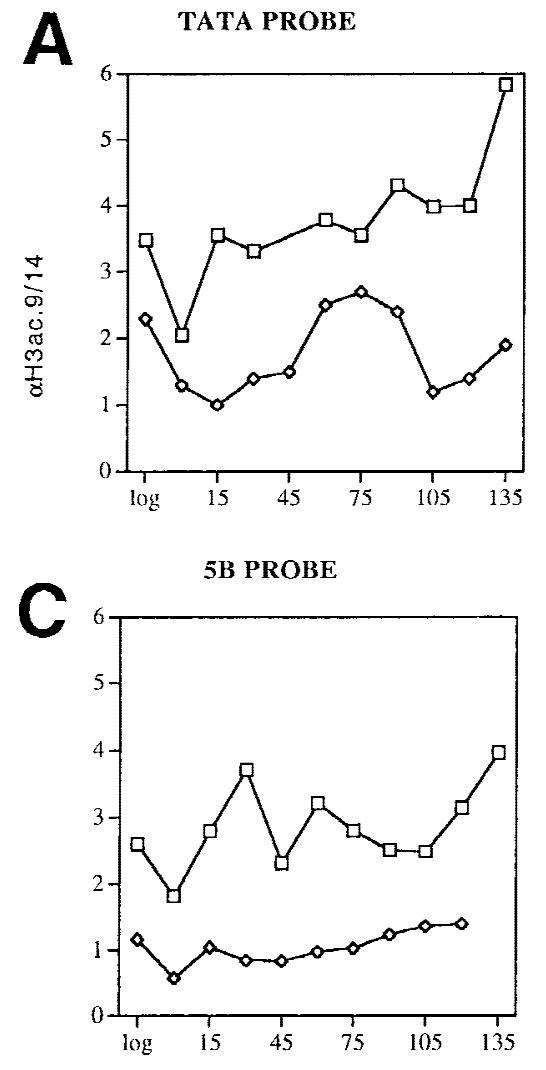
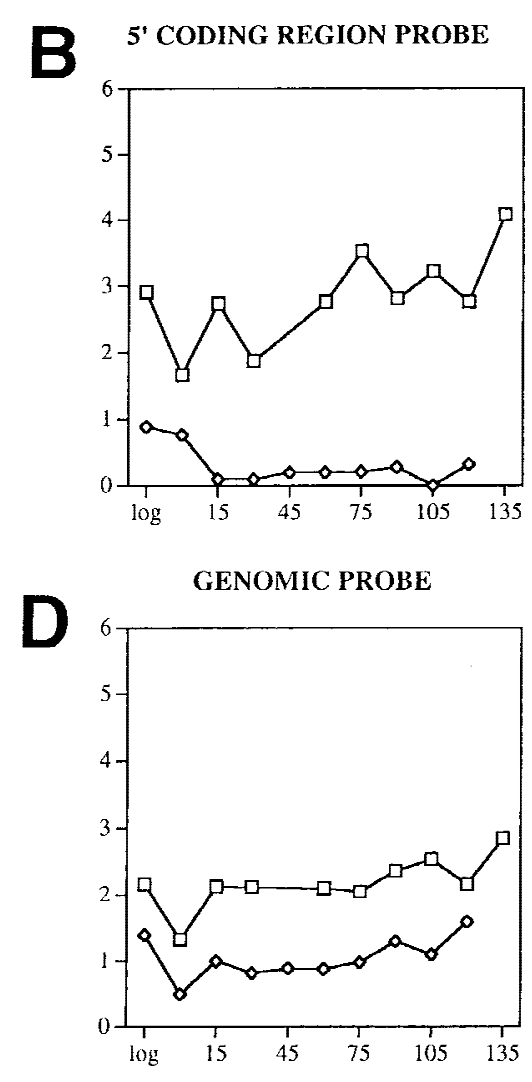

GENOMIC PROBE

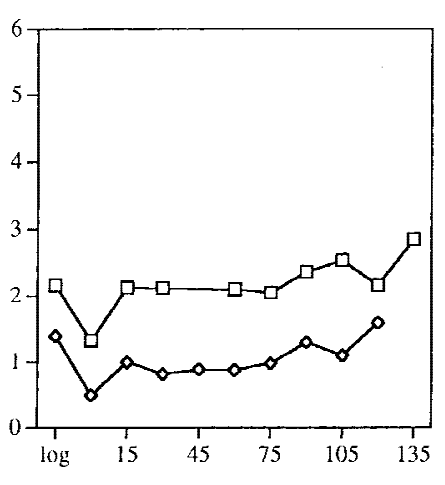

Figure 4. The entire $H O$ gene is acetylated throughout the cell cycle in $\sin 3 \Delta$ cells. $\sin 3^{-}$ cells were synchronized with nocodazole and samples were fixed for chromatin IP after synchronous release. ChIPs were performed with the antibody to diacetylated histone $\mathrm{H} 3$ (ac.9/ 14). In each panel, data from $\sin 3$ cells $(\square)$ are compared with wild-type $(\diamond)$. $(A)$ Acetylation at the HO TATA region in wild-type cells and in $\sin 3$ mutants. (B) Acetylation at the $5^{\prime}$ end of the $H O$ coding region in wild-type cells and in $\sin 3$ mutants. (C) Acetylation at the upstream Swi5pbinding site in wild-type cells and in sin3 mutants. $(D)$ Genomic H3 acetylation in wild-type and $\sin 3$ cells. 


\section{Discussion}

We have shown here that there is a cell cycle-regulated acetylation of histones $\mathrm{H} 3$ and $\mathrm{H} 4$ that occurs throughout a $1000-b p$ region of the HO promoter. These acetylation events require the Gcn5p HAT, a site-specific activator, Swi5p, and the ATP-dependent chromatin remodeler, SWI/SNF. In contrast, cell cycle-regulated acetylation does not require the Swi4p/Swi6p activator and it precedes, and is independent of, transcription. Furthermore, inactivation of SIN3 leads to high levels of histone acetylation at the $\mathrm{HO}$ locus throughout the cell cycle, suggesting that the $\operatorname{Sin} 3 p / R p d 3 p$ complex may control deacetylation events at the $H O$ promoter. Our studies are consistent with a sequential model for activation of $H O$ transcription that involves at least two functionally dependent chromatin remodeling events.

The first step in the activation of $H O$ transcription is likely to involve the Swi5p activator. Swi5p is expressed in S phase, in which it remains localized in the cytoplasm until it enters the nucleus in late anaphase after inactivation of a Clbp/Cdc28p kinase (Moll et al. 1991; Tebb et al. 1993). After its arrival in the nucleus, Swi5p is competent for DNA binding and transcriptional activation, because SWI5 is required for expression of a number of genes in early $\mathrm{G}_{1}$, such as ASH1 (Bobola et al. 1996), SIC1 (Knapp et al. 1996; Toyn et al. 1997), EGT2 (Kovacech et al. 1996), CDC6 (Piatti et al. 1995), PCL2 and PCL9 (Aerne et al. 1998). Although Swi5p probably binds in early $\mathrm{G}_{1}$ to its two sites far upstream of the $H O$ TATA element (see Fig. 2A), HO transcription does not ensue until much later at the $\mathrm{G}_{1} / \mathrm{S}$ boundary. Swi5p may carry out its function prior to the actual activation of $H O$ transcription, because Swi5p is rapidly degraded during $\mathrm{G}_{1}$ (Tebb et al. 1993). Because Swi5p is required for GCN5-dependent acetylation of nucleosomes that encompass the downstream cell cycle boxes, this early function may involve the recruitment or activation of a Gcn5p-containing HAT complex.

Our data also indicate that SWI/SNF acts at an early step in $\mathrm{HO}$ expression, as it is required for the recruitment or activity of a GCN5-dependent acetyltransferase in mid- $G_{1}$. Although our acetylation data cannot define an order of action for Swi5p and SWI/SNF, Nasmyth and colleagues (Cosma et al. 1999) have used a ChIP assay to show that Swi5p associates with the HO locus prior to SWI/SNF, and that Swi5p is required for subsequent SWI/SNF recruitment. Once SWI/SNF is recruited by Swi5p, our data suggests that remodeling by SWI/SNF is required for the recruitment or activity of a GCN5-dependent HAT.

In mid- $\mathrm{G}_{1}$, nucleosomes within a $\sim 1-\mathrm{kb}$ segment of the $H O$ upstream regulatory region are acetylated by a GCN5-dependent HAT complex. We propose that this HAT complex is targeted to the promoter either directly by interaction with Swi5p, or as a result of SWI/SNF remodeling. Acetylation appears to initiate at the distal SCB elements and then continues into the TATA region, consistent with a movement of the acetyltransferase complex unidirectionally along the promoter. We pro- pose that these acetylation events are required to facilitate the binding of Swi4p/Swi6p to its binding sites located within this domain. SWI/SNF may also be required for this step, because SWI/SNF is required for the UAS activity of this SWI4/SWI6-dependent UAS (Nasmyth 1987; Sternberg et al. 1987). Once Swi4p/Swi6p are bound to the $H O$ upstream region, transcription can only be activated at the $\mathrm{G}_{1} / \mathrm{S}$ boundary when a Cdc28p/Clnp kinase directly or indirectly stimulates Swi4p/Swi6p activity. We anticipate that this last step may involve the actual recruitment of the general transcription machinery or RNA polymerase II holoenzyme.

\section{Role of histone deacetylases in the regulation of $\mathrm{HO}$ expression}

Whereas Swi5p and SWI/SNF are required to establish a domain of histone acetylation in mid-G1, an intact $\operatorname{Sin} 3 p / R p d 3 p$ deacetylase complex is required to erase these acetylation events in early $S$ phase and to ensure that acetylation is low as cells enter the subsequent cell cycle. Consequently, in a sin3 deletion mutant, the $H O$ locus is encompassed by highly acetylated nucleosomes throughout the cell cycle. What are the functional consequences of constitutive histone acetylation? Inactivation of the Sin3p/Rpd3p complex allows $H O$ expression in the absence of SWI5, SWI/SNF, or GCN5; however, sin3 or rpd3 mutants still require SWI4 and SWI6 (Nasmyth et al. 1987; Sternberg et al. 1987; Stillman 1994; Wang et al. 1994; Perez-Martin and Johnson 1997). Furthermore, if a daughter cell inherits a highly acetylated $H O$ locus, the Ashlp protein is unable to repress transcription in $\mathrm{G}_{1}$. Consequently, $\mathrm{HO}$ is expressed in both mother and daughter cells in sin3 mutants. (Nasmyth et al. 1987; Sternberg et al. 1987). This suggests that deacetylation of the $H O$ locus is normally required for repression of $\mathrm{HO}$ by Ashlp in daughter cells.

Regulation of histone acetylation also appears to be required for control of $\mathrm{HO}$ expression during the mating process. If MATa cells are exposed to the mating pheromone, $\alpha$ factor, the Cdc28p/Clnp kinase is inactivated, cells arrest in the $\mathrm{G}_{1} / \mathrm{S}$ phase of the cell cycle, and $H O$ is not expressed. When wild-type cells are released from the $\alpha$ factor block, $H O$ is not expressed until the next cell cycle. The repression of $H O$ in the first cell cycle following release probably ensures that the MATa cell has one additional opportunity to mate with a nearby MAT $\alpha$ partner before the mating type is switched by the $\mathrm{HO}$ endonuclease in the next cell cycle. However, in the absence of a functional $\operatorname{Sin} 3 \mathrm{p} / \mathrm{Rpd} 3 \mathrm{p}$ deacetylase complex, HO is expressed immediately after release from the $\alpha$ factor block (Nasmyth et al. 1987). Thus, the functional interplay between histone acetylases (e.g., Gcn5p) and deacetylases (e.g., $\mathrm{Rpd} 3$ ) is crucial for several developmental controls of $\mathrm{HO}$ expression.

Another puzzling question concerns how the size of the acetylated histone domain is controlled. Previous studies have suggested that acetylation (Kuo et al. 1998) or deacetylation (Kadosh and Struhl 1998) might be limited to a region of only one or two nucleosomes. In these 
cases, a HAT or HDAC might be targeted to a single site, in which it can only act on nucleosomes within reach. However, at $H O$, a GCN5-dependent complex acetylates perhaps six or seven nucleosomes, and the cell cycle timing of acetylation at different positions in the promoter suggests that Gcn5p might be able to move from its targeting site. Furthermore, we find that acetylation does not extend upstream of the SCB elements or downstream of the TATA region. In the latter case, the 5' ORF probe does not detect a $G_{1}$ peak of acetylation even though it is only $200 \mathrm{bp}$ from the TATA region in which the strong $\mathrm{G}_{1}$ peak is detected. In fact, $\mathrm{H} 3$ acetylation at the $\mathrm{HO}$ coding region is much less than the level observed throughout the genome (cf. Figs. 1C and 2B). We believe it is unlikely that Swi5p or Swi4/Swi6p activators directly contribute to establishing the size of the acetylated domain, because the $\sim 1-\mathrm{kb}$ domain is established in a swi4 deletion mutant, and Swi5p does not appear to remain stably associated with the $H O$ locus during $G_{1}$ (Cosma et al. 1999). We favor a model in which boundary elements might exist in the $H O$ promoter that either sequester a deacetylase or prevent the escape of a Gcn5pcontaining HAT from the desired target region. One type of boundary might consist of TFIID, bound to the TATA element, which might restrain the Gen5p-containing SAGA complex via physical interactions with one or more SPT subunits. Alternatively, a recent study (Pikaart et al. 1998) proposed that one possible function of insulator elements is to sequester acetylases or to exclude deacetylases. Consistent with this model, we have shown here that inactivation of the $\operatorname{Sin} 3 p / R p d 3 p$ complex leds to an expanded domain of $\mathrm{H} 3$ acetylation that encompasses the entire $\mathrm{HO}$ locus.

\section{Dependent chromatin remodeling events}

Many of the genes whose expression requires SWI/SNF also rely on the activity of the Gen5p HAT (Pollard and Peterson 1997). Previously, we proposed that Gcn5p acetyltransferase activity might control the ability of SWI/SNF to function on compacted nucleosomal arrays (Pollard and Peterson 1998). However, we have shown here that at the HO locus, SWI/SNF appears to be required at an early step that facilitates the recruitment or activity of the Gcn5p-containing HAT complex. It also possible that SWI/SNF functions at more than one step during the activation of $H O$. For instance, SWI/SNF may facilitate the binding of Swi4p/Swi6p to acetylated, nucleosomal sites. In this case, the prior acetylation of nucleosomes that encompass the Swi4p/Swi6p-binding sites may control the activity of SWI/SNF. In support of this view, the histone amino-terminal domains and the state of histone acetylation does alter the ability of SWI/ SNF to function catalytically in vitro (Logie et al. 1999). Thus, although our analyses of the transitions in $\mathrm{HO}$ chromatin structure have uncovered one functional coupling between two distinct remodeling enzymes, it seems likely that the cell will have evolved multiple scenarios to ensure precise transcriptional regulation.

\section{Materials and methods}

Strains and medium

Strains were grown in YEP medium $(2 \%$ yeast extract, $1 \%$ bactopeptone) containing either $2 \%$ glucose or $2 \%$ galactose. Most yeast strains used in this study are isogenic derivatives of the YPH strain set described by Sikorski and Hieter (1989). Deletion of ASH1 was accomplished by a one-step gene disruption and was verified by Southern blot. Our isogenic set has the follow-

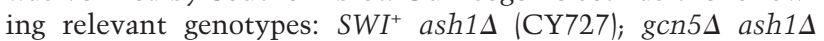

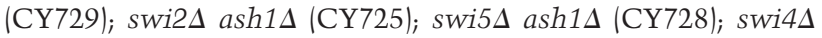
ash1s (CY726); $\sin 3 \Delta$ ash1s (CY755).

\section{Cell cycle synchronization and ChIPs}

To arrest cells in $\mathrm{G}_{2} / \mathrm{M}$, nocodazole (Sigma, $15 \mu \mathrm{g} / \mathrm{ml}$ in DMSO) was added directly to the medium to a final concentration of 15 $\mu \mathrm{g} / \mathrm{ml}$. Cells were arrested for $\sim 2 \mathrm{hr}$ until arrest was at least $90 \%$ complete as determined by examination under the microscope. Cells were pelleted and washed in water, then resuspended in nocodazole-free medium. Samples were taken every $15 \mathrm{~min}$ and cross-linked with $1 \%$ formaldehyde for $15 \mathrm{~min}$ at room temperature. Cross-linking was quenched with $125 \mathrm{~mm}$ glycine and whole cell extracts were prepared for use in ChIPs. ChIPs were performed as described previously (Strahl-Bolsinger et al. 1997; Kuo et al. 1998). The average size of fragmented chromatin was $\sim 500 \mathrm{bp}$. All ChIP experiments presented in this manuscript were performed at least four times-immunoprecipitations were performed two times for each of two independent cell synchronizations.

\section{Probes and RNA analysis}

All DNA hybridization probes used in these studies were generated by PCR and labeled by random priming. The probes (with their endpoints shown in parentheses relative to the start site of $H O$ transcription) were amplified with the following primers: HO TATA (-227:-25), 5'-gcgtcacgaaaaaagaaatcaatcctacacagg-3' and $3^{\prime}$-cgagatttaggtataggagtattcgtcg- $5^{\prime} ;$ SCB1 (-950:-757), $5^{\prime}$ ccaaacttattacttttcctatttgaggttgg- $3^{\prime}$ and $3^{\prime}$-gcttttcactagagctatgttaatctcctagg5', SCB2 (-659:-461), 5'-gcgtcacgaaaaaagaaatcaatcctacacagg- $3^{\prime}$ and $3^{\prime}$-ggacaaagatacggaaagagaatcattaagtgc-5', 5A (-1386:-1144), 5'-ccttttttcatttttccetacgctcagggc-3' and $3^{\prime}$ cccggcttagcgcatttttcaaactaagcacc-5'; 5B (-1847:-1637), 5'-cgtaaaattgtgcctttggacttaaaatggcg- $3^{\prime}$ and $3^{\prime}$-ccattagaaacaatccacgctaaaataggc-5'; $3^{\prime}$ ORF $(+720:+947)$, 5'-ggagagtttaagagaaaatgcgaaaatctgg- $3^{\prime}$ and $3^{\prime}$-cctctcttcgtttagggacttaaatacagcc-5'; 5'ORF $(+3:+233), 5^{\prime}$-gctttctgaaaacacgactattctgatggc- $3^{\prime}$ and $3^{\prime}$-gggtccgcatcttgtcaaatagtcgcagaacg-5'. The GCS1 probe was amplified with $5^{\prime}$-ggaaagttcaattcacattgtagctataatgg- $3^{\prime}$ and $3^{\prime}$-gggtagccgcaacaacatgacaacgttccg-5'; and the SSB1 probe was amplified with $5^{\prime}$-ccatttttagactttttcttaactagaatgc- $3^{\prime}$ and $3^{\prime}$-gcgetttctttttgtttataaaattaacac-5'.

HO transcription was detected by primer extension. Total RNA was prepared from cells by a glass bead lysis and phenol extraction method (Peterson and Herskowitz 1992). A total of $10 \mu \mathrm{g}$ of RNA was hybridized with $1 \mathrm{ng}$ of kinase-labeled oligonucleotide in $250 \mathrm{mM} \mathrm{KCl}$ at $50^{\circ} \mathrm{C}$ for $1 \mathrm{hr}$. Primers were extended with AMV reverse transcriptase (Promega) in $50 \mathrm{~mm}$ Tris ( $\mathrm{pH} 8.0$ ), $5 \mathrm{~mm} \mathrm{MgCl}, 100 \mu \mathrm{g} / \mathrm{ml}$ actinomycin $\mathrm{D}, 10 \mathrm{~mm}$ DTT, $25 \mathrm{~mm}$ EDTA, and $0.25 \mathrm{~mm}$ dNTPs for $30 \mathrm{~min}$ at $42^{\circ} \mathrm{C}$. Extension products were separated on an $8 \%$ acrylamide $/ 8 \mathrm{M}$ urea denaturing gel, and gels were fixed with $5 \% \mathrm{TCA}$, dried, and exposed to X-ray film. 


\section{Acknowledgments}

We thank Kim Nasmyth for providing strains and for sharing unpublished results, Anita Sil and Ira Herskowitz for providing the ASH1 knockout plasmids, and Colin Logie, Mike Samuels, and other members of the Peterson laboratory for critical reading of the manuscript. This research was supported by grants from the National Institutes of Health (GM49650) to C.L.P. and the American Cancer Society to J.E.K. C.L.P. is a Scholar of the Leukemia Society of America.

The publication costs of this article were defrayed in part by payment of page charges. This article must therefore be hereby marked 'advertisement' in accordance with 18 USC section 1734 solely to indicate this fact.

\section{References}

Aerne, B.L., A.L. Johnson, J.H. Toyn, and L.H. Johnston. 1998. Swi5 controls a novel wave of cyclin synthesis in late mitosis. Mol. Biol. Cell. 9: 945-956.

Amon, A., M. Tyers, B. Futcher, and K. Nasmyth. 1993. Mechanisms that help the yeast cell cycle clock tick: G2 cyclins transcriptionally activate G2 cyclins and repress G1 cyclins. Cell 74: 993-1007.

Bobola, N., R.P. Jansen, T.H. Shin, and K. Nasmyth. 1996. Asymmetric accumulation of Ash1p in postanaphase nuclei depends on a myosin and restricts yeast mating-type switching to mother cells. Cell 84: 699-709.

Breeden, L. and K. Nasmyth. 1987. Cell cycle control of the yeast $\mathrm{HO}$ gene: Cis- and trans-acting regulators. Cell 48: 389-397.

Brownell, J.E., J. Zhou, T. Ranalli, R. Kobayashi, D.G. Edmondson, S.Y. Roth, and C.D. Allis. 1996. Tetrahymena histone acetyltransferase A: A homolog to yeast Gcn5p linking histone acetylation to gene activation. Cell 84: 843-851.

Burns, L.G. and C.L. Peterson. 1997. The yeast SWI-SNF complex facilitates binding of a transcriptional activator to nucleosomal sites in vivo. Mol. Cell. Biol. 17: 4811-4819.

Candau, R., J. Zhou, C.D. Allis, and S.L. Berger. 1997. Histone acetyltransferase activity and interaction with ADA2 are critical for Gen5p function in vivo. EMBO J. 16: 555-565.

Cosma, M.P., T. Tanaka, and K. Nasmyth. 1999. Ordered recruitment of transcription and chromatin re-modeling factors to a cell cycle and developmentally regulated promoter. Cell 978: 299-311.

Cote, J., J. Quinn, J.L. Workman, and C.L. Peterson. 1994. Stimulation of GAL4 derivative binding to nucleosomal DNA by the yeast SWI/SNF complex. Science 265: 53-60.

Cote, J., C.L. Peterson, and J.L. Workman. 1998. Perturbation of nucleosome core structure by the SWI/SNF complex persists after its detachment, enhancing subsequent transcription factor binding. Proc. Natl. Acad. Sci. 95: 4947-4952.

Eberharter, A., S. John, P.A. Grant, R.T. Utley, and J.L. Workman. 1998. Identification and analysis of yeast nucleosomal histone acetyltransferase complexes. Methods 15: 315-321.

Grant, P.A., L. Duggan, J. Cote, S.M. Roberts, J.E. Brownell, R. Candau, R. Ohba, T. Owen-Hughes, C.D. Allis, F. Winston, S.L. Berger, and J.L. Workman. 1997. Yeast Gen5 functions in two multisubunit complexes to acetylate nucleosomal histones: characterization of an Ada complex and the SAGA (Spt/Ada) complex. Genes \& Dev. 11: 1640-1650.

Grant, P.A., D. Schieltz, M.G. Pray-Grant, D.J. Steger, J.C. Reese, J.R. Yates, 3rd, and J.L. Workman. 1998. A subset of TAF(II)s are integral components of the SAGA complex required for nucleosome acetylation and transcriptional stimulation. Cell 94: 45-53.
Harrington, L.A. and B.J. Andrews. 1996. Binding to the yeast Swi4,6-dependent cell cycle box, CACGAAA, is cell cycle regulated in vivo. Nucleic Acids Res. 24: 558-565.

Herskowitz, I., B. Andrews, W. Kruger, J. Ogas, A. Sil, C. Coburn, and C. Peterson. 1992. Integration of multiple regulatory inputs in the control of $\mathrm{HO}$ expression in yeast. In Transcriptional regulation (ed. S. McKnight adn K. Yamamoto) pp. 949-974. Cold Spring Harbor Laboratory Press, Cold Spring Harbor, NY.

Ikeda, K., D.J. Steger, A. Eberharter, and J.L. Workman. 1999. Activation domain-specific and general transcription stimulation by native histone acetyltransferase complexes. Mol. Cell. Biol. 19: 855-863.

Imbalzano, A.N. 1998. SWI/SNF complexes and facilitation of TATA binding protein:nucleosome interactions. Methods 15: 303-314.

Imbalzano, A.N., H. Kwon, M.R. Green, and R.E. Kingston. 1994. Facilitated binding of TATA-binding protein to nucleosomal DNA. Nature 370: 481-485.

Jansen, R.P., C. Dowzer, C. Michaelis, M. Galova, and K. Nasmyth. 1996. Mother cell-specific HO expression in budding yeast depends on the unconventional myosin myo4p and other cytoplasmic proteins. Cell 84: 687-697.

Kadosh, D. and K. Struhl. 1998. Targeted recruitment of the Sin3-Rpd3 histone deacetylase complex generates a highly localized domain of repressed chromatin in vivo. Mol. Cell. Biol. 18: 5121-5127.

Kasten, M.M., S. Dorland, and D.J. Stillman. 1997. A large protein complex containing the yeast $\operatorname{Sin} 3 \mathrm{p}$ and Rpd 3 transcriptional regulators. Mol. Cell. Biol. 17: 4852-4858.

Knapp, D., L. Bhoite, D.J. Stillman, and K. Nasmyth. 1996. The transcription factor Swi5 regulates expression of the cyclin kinase inhibitor p40SIC1. Mol. Cell. Biol. 16: 5701-5707.

Koch, C., A. Schleiffer, G. Ammerer, and K. Nasmyth. 1996. Switching transcription on and off during the yeast cell cycle: Cln/Cdc28 kinases activate bound transcription factor SBF (Swi4/Swi6) at start, whereas $\mathrm{Clb} / \mathrm{Cdc} 28$ kinases displace it from the promoter in $\mathrm{G}_{2}$. Genes \& Dev. 10: 129141.

Kovacech, B., K. Nasmyth, and T. Schuster. 1996. EGT2 gene transcription is induced predominantly by Swi5 in early G1. Mol. Cell. Biol. 16: 3264-3274.

Kuo, M.-H., J.E. Brownell, R.E. Sobel, T.A. Ranalli, R.C. Cook, D.G. Edmondson, S.Y. Roth, and C.D. Allis. 1996. Transcription-linked acetylation by Gen5p of histones $\mathrm{H} 3$ and $\mathrm{H} 4$ at specific lysines. Nature 383: 269-272.

Kuo, M.H., J. Zhou, P. Jambeck, M.E. Churchill, and C.D. Allis. 1998. Histone acetyltransferase activity of yeast Gcn5p is required for the activation of target genes in vivo. Genes \& Dev. 12: 627-639.

Laurent, B.C. and M. Carlson. 1992. Yeast SNF2/SWI2, SNF5, and SNF6 proteins function coordinately with the gene-specific transcriptional activators GAL4 and Bicoid. Genes \& Dev. 6 1707-1715.

Laurent, B.C., I. Treich, and M. Carlson. 1993. Role of yeast SNF and SWI proteins in transcriptional activation. Cold Spring Harb. Symp. Quant. Biol. 58: 257-263.

Logie, C. and C.L. Peterson. 1997. Catalytic activity of the yeast SWI/SNF complex on reconstituted nucleosome arrays. EMBO J. 16: 6772-6782.

Logie, C., C. Tse, J.C. Hansen, and C.L. Peterson. 1999. The core histone $\mathrm{N}$-terminal domains are required for multiple rounds of catalytic chromatin remodeling by the SWI/SNF and RSC complexes. Biochemistry 38: 2514-2522.

Moll, T., G. Tebb, U. Surana, H. Robitsch, and K. Nasmyth. 1991. The role of phosphorylation and the CDC28 protein 
kinase in cell cycle- regulated nuclear import of the S. cerevisiae transcription factor SWI5. Cell 66: 743-758.

Nasmyth, K. 1987. The determination of mother cell-specific mating type switching in yeast by a specific regulator of $H O$ transcription. EMBO J. 6: 243-248.

- 1993. Regulating the HO endonuclease in yeast. Curr. Opin. Gen. Dev. 3: 286-294.

Nasmyth, K., D. Stillman, and D. Kipling. 1987. Both positive and negative regulators of $\mathrm{HO}$ transcription are required for mother-cell-specific mating-type switching in yeast. Cell 48: $579-587$.

Perez-Martin, J. and A.D. Johnson. 1998a. The C-terminal domain of Sin 1 interacts with the SWI-SNF complex in yeast. Mol. Cell. Biol. 18: 4157-4164.

1998b. Mutations in chromatin components suppress a defect of Gcn5 protein in Saccharomyces cerevisiae. Mol. Cell. Biol. 18: 1049-1054.

Peterson, C.L. 1996. Multiple SWItches to turn on chromatin? Curr. Opin. Gen. Dev. 6: 171-175.

Peterson, C.L. and I. Herskowitz. 1992. Characterization of the yeast SWI1, SWI2, and SWI3 genes, which encode a global activator of transcription. Cell 68: 573-583.

Peterson, C.L., A. Dingwall, and M.P. Scott. 1994. Five SWI/ SNF gene products are components of a large multisubunit complex required for transcription enhancement. Proc. Natl. Acad. Sci. 91: 2905-2908.

Piatti, S., C. Lengauer, and K. Nasmyth. 1995. Cdc6 is an unstable protein whose de novo synthesis in G1 is important for the onset of S phase and for preventing a 'reductional' anaphase in the budding yeast Saccharomyces cerevisiae. EMBO I. 14: 3788-3799.

Pikaart, M J., F. Recillas-Targa, and G. Felsenfeld. 1998. Loss of transcriptional activity of a transgene is accompanied by DNA methylation and histone deacetylation and is prevented by insulators. Genes \& Dev. 12: 2852-2862.

Pollard, K.J. and C.L. Peterson. 1997. Role for ADA/Gen5p products in antagonizing chromatin-mediated transcriptional repression. Mol. Cell. Biol. 17: 6212-6222.

1998. Chromatin remodeling: A marriage between two families? BioEssays 20: 771-780.

Roberts, S.M. and F. Winston. 1997. Essential functional interactions of SAGA, a Saccharomyces cerevisiae complex of Spt, Ada, and Gen5 proteins, with the Snf/Swi and Srb/mediator complexes. Genetics 147: 451-465.

Ryan, M.P., R. Jones, and R.H. Morse. 1998. SWI-SNF complex participation in transcriptional activation at a step subsequent to activator binding. Mol. Cell. Biol. 18: 1774-1782.

Saleh, A., V. Lang, R. Cook, and C.J. Brandl. 1997. Identification of native complexes containing the yeast coactivator/repressor proteins NGG1/ADA3 and ADA2. I. Biol. Chem. 272: 5571-5578.

Sikorski, R.S. and P. Hieter. 1989. A system of shuttle vectors and yeast host strains designed for efficient manipulation of DNA in Saccharomyces cerevisiae. Genetics 122: 19-27.

Sil, A. and I. Herskowitz. 1996. Identification of an asymmetrically localized determinant, Ash1p, required for lineage-specific transcription of the yeast HO gene. Cell 84: 711-722.

Steger, D.J., A. Eberharter, S. John, P.A. Grant, and J.L. Workman. 1998. Purified histone acetyltransferase complexes stimulate HIV-1 transcription from preassembled nucleosomal arrays. Proc. Nat1. Acad. Sci. 95: 12924-12929.

Stern, M., R.E. Jensen, and I. Herskowitz. 1984. Five SWI genes are required for the expression of the $H O$ gene in yeast. $J$. Mol. Biol. 178: 853-868.

Sternberg, P.W., M.J. Stern, I. Clark, and I. Herskowitz. 1987. Activation of the yeast $\mathrm{HO}$ gene by release from multiple negative controls. Cell 48: 567-577.

Sterner, D.E., P.A. Grant, S.M. Roberts, L.J. Duggan, R. Belotserkovskaya, L.A. Pacella, F. Winston, J.L. Workman, and S.L. Berger. 1999. Functional organization of the yeast SAGA complex: Distinct components involved in structural integrity, nucleosome acetylation, and TATA- binding protein interaction. Mol. Cell. Biol. 19: 86-98.

Stillman, D.J., S. Dorland, and Y. Yu. 1994. Epistasis analysis of suppressor mutations that allow HO expression in the absence of the yeast Swi5p transcriptional activator. Genetics 146: 781-788.

Strahl-Bolsinger, S., A. Hecht, K. Luo, and M. Grunstein. 1997. SIR2 and SIR4 interactions differ in core and extended telomeric heterochromatin in yeast. Genes \& Dev. 11: 83-93.

Tebb, G., T. Moll, C. Dowzer, and K. Nasmyth. 1993. Swi5p instability may be necessary but is not sufficient for asymmetric HO expression in yeast. Genes \& Dev. 7: 517-528.

Toyn, J.H., A.L. Johnson, J.D. Donovan, W.M. Toone, and L.H. Johnston. 1997. The Swi5 transcription factor of Saccharomyces cerevisiae has a role in exit from mitosis through induction of the cdk-inhibitor Sicl in telophase. Genetics 145: 85-96.

Utley, R.T., J. Cote, T. Owen-Hughes, and J.L. Workman. 1997. SWI/SNF stimulates the formation of disparate activatornucleosome complexes but is partially redundant with cooperative binding. J. Biol. Chem. 272: 12642-12649.

Utley, R.T., K. Ikeda, P.A. Grant, J. Cote, D.J. Steger, A. Eberharter, S. John, and J.L. Workman. 1998. Transcriptional activators direct histone acetyltransferase complexes to nucleosomes. Nature 394: 498-502.

Wang, H., L. Reynolds-Hager, and D.J. Stillman. 1994. Genetic interactions between SIN3 mutations and the Saccharomyces cerevisiae transcriptional activators encoded by MCM1, STE12, and SWI1. Mol. \& Gen. Genet. 245: 675-685.

Wang, L., L. Liu, and S.L. Berger. 1998. Critical residues for histone acetylation by Gen5, functioning in Ada and SAGA complexes, are also required for transcriptional function in vivo. Genes \& Dev. 12: 640-653.

Workman, J.L. and R.E. Kingston. 1998. Alteration of nucleosome structure as a mechanism of transcriptional regulation. Annu. Rev. Biochem. 67: 545-579.

Zhang, W., J.R. Bone, D.G. Edmondson, B.M. Turner, and S.Y. Roth. 1998. Essential and redundant functions of histone acetylation revealed by mutation of target lysines and loss of the Gcn5p acetyltransferase. EMBO J. 17: 3155-3167. 


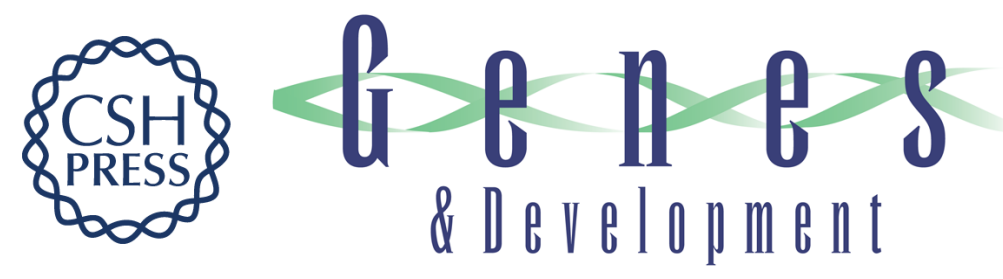

\section{Cell cycle-regulated histone acetylation required for expression of the yeast $\mathrm{HO}$ gene}

Jocelyn E. Krebs, Min-Hao Kuo, C. David Allis, et al.

Genes Dev. 1999, 13:

References This article cites 60 articles, 34 of which can be accessed free at:

http://genesdev.cshlp.org/content/13/11/1412.full.html\#ref-list-1

License

Email Alerting
Service

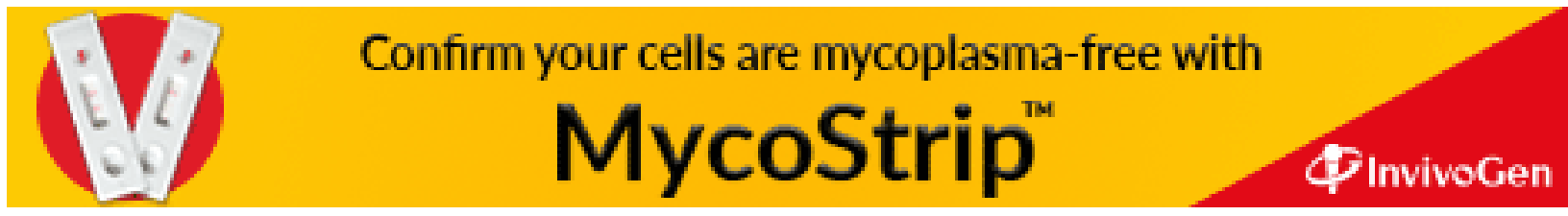

